\title{
Ultraviolet radiation accelerates BRAF-driven melanomagenesis by targeting TP53
}

\author{
Amaya Viros ${ }^{\# 1}$, Berta Sanchez-Laorden ${ }^{\# 1}$, Malin Pedersen ${ }^{\# 2}$, Simon J. Furney ${ }^{\# 1}$, Joel Rae ${ }^{2}$, \\ Kate Hogan ${ }^{1}$, Sarah Ejiama ${ }^{1}$, Maria Romina Girotti ${ }^{1}$, Martin Cook $^{1,3}$, Nathalie Dhomen ${ }^{2}$, and \\ Richard Marais ${ }^{1,2,4}$ \\ ${ }^{1}$ Molecular Oncology Group, Cancer Research UK Manchester Institute, The University of \\ Manchester, Wilmslow Road, Manchester, M20 4BX, UK \\ ${ }^{2}$ Signal Transduction Team, The Institute of Cancer Research, 237 Fulham Road, London, SW3 \\ 6JB, UK \\ ${ }^{3}$ Histopathology, Royal Surrey County Hospital, Egerton Road, Guildford, GU2 7XX UK \\ \# These authors contributed equally to this work.
}

\section{Abstract}

Cutaneous melanoma is epidemiologically linked to ultraviolet radiation (UVR), but the molecular mechanisms by which UVR drives melanomagenesis remain unclear ${ }^{1,2}$. The most common somatic mutation in melanoma is a V600E substitution in BRAF, which is an early event ${ }^{3}$. To investigate how UVR accelerates oncogenic BRAF-driven melanomagenesis, we used a ${ }^{\mathrm{V} 600} \mathrm{E}$ BRAF mouse model. In mice expressing ${ }^{\mathrm{V} 600 \mathrm{E}_{B R}} \mathrm{BF}$ in their melanocytes, a single dose of UVR that mimicked mild sunburn in humans induced clonal expansion of the melanocytes, and repeated doses of UVR increased melanoma burden. We show that sunscreen (UVA superior: UVB SPF50) delayed the onset of UVR-driven melanoma, but only provided partial protection. The UVR-exposed tumours presented increased numbers of single nucleotide variants (SNVs) and we observed mutations (H39Y, S124F, R245C, R270C, C272G) in the Trp53 tumour suppressor in $\sim 40 \%$ of cases. TP53 is an accepted UVR target in non-melanoma skin cancer, but is not thought to play a major role in melanoma ${ }^{4}$. However, we show that mutant $\operatorname{Trp} 53$ accelerated ${ }^{\mathrm{V} 600 \mathrm{E}} \mathrm{BRAF}$-driven melanomagenesis and that TP53 mutations are linked to evidence of UVR-induced DNA damage in human melanoma. Thus, we provide mechanistic insight into epidemiological data linking UVR to acquired naevi in humans ${ }^{5}$. We identify TP53/Trp53 as a UVR-target gene that cooperates with ${ }^{\mathrm{V} 600 \mathrm{E}} \mathrm{BRAF}$ to induce melanoma, providing molecular

Users may view, print, copy, and download text and data-mine the content in such documents, for the purposes of academic research, subject always to the full Conditions of use:http://www.nature.com/authors/editorial_policies/license.html\#terms

${ }^{4}$ Corresponding Author: Richard Marais PhD., Cancer Research UK Manchester Institute, Wilmslow Road, Manchester, M20 4BX, UK, Tel: +44 (0)161446 3100, richard.marais@cruk.manchester.ac.uk.

AUTHOR CONTRIBUTIONS.

A.V., B.S-L. and R.M. designed the study, analysed the data and wrote the paper. M.P. designed and performed experiments and analysed data. S.J.F. designed and performed bioinformatics analysis and analysed data. K.H., J.R., M.R.G., M.C. and N.D. performed experiments. S.E. validated WES SNVs.

The authors declare no competing financial interests.

Exome sequence and array CGH data have been deposited at the European Genome-Phenome Archive (http://www.ebi.ac.uk/ega/), which is hosted by the EBI, under accession numbers EGAS00001000729 and EGAS00001000735. 
insight into how UVR accelerates melanomagenesis. Our study validates public health campaigns that promote sunscreen protection for individuals at risk of melanoma.

We expressed ${ }^{\mathrm{V} 600 \mathrm{E}} \mathrm{BRAF}$ in the melanocytes of 2-month old mice ${ }^{6}$ and one month later, protected half of the shaved backs of the mice with a cloth and exposed the other half to low doses of UVR (Fig 1a, Extended Data Fig. 1a, 1b). UVR upregulates Trp537, and within 24h of a single UVR exposure we observed Trp53 staining in $41 \%$ of epidermal keratinocytes and down into the reticular dermis (Fig. 1b; Extended Data Fig. 1c, Table 1). We also observed abundant sunburn cells $(\sim 17$ cells/mm) in the basal layer of the UVR-exposed skin (Fig. 1c; Extended Data Table 1). The skin did not blister, but after 24-48 hours it developed mild erythema, and after seven days it was rough to the touch, presented fine desquamation and was thickened due to hyperkeratosis, epidermal hypertrophy and a thickened fibrotic dermis (Fig. 1d).

UVR induces melanocyte proliferation ${ }^{8}$, and naevogenesis is driven by clonal expansion of melanocytes expressing mutant $B R A F$, the acquisition of which is an early event ${ }^{3}$. Furthermore, the incidence of acquired human naevi, $\sim 80 \%$ of which carry $B R A F$ mutations ${ }^{9}$, is linked to UVR exposure ${ }^{10}$. We show that UVR induced ${ }^{\mathrm{V} 600 \mathrm{E}} \mathrm{BRAF}$ melanocyte proliferation in vivo (Extended Data Fig. 2) and within 7 days, the UVRexposed skin presented more abundant and larger naevi (Fig. 1d; Extended Data Fig. 3a-d; Extended Data Table 2). These UVR-induced changes persisted (Fig. 1e) and the UVRexposed skin darkened (Fig. 1f). UVR did not induce naevi or skin darkening in non- ${ }^{\mathrm{V} 600 \mathrm{E}}$ BRAF mice (Extended Data Fig. 3e, 3f). Thus, we validate human epidemiological studies by showing that ${ }^{\mathrm{V} 600 \mathrm{E}} \mathrm{BRAF}$-expressing melanocytes are susceptible to proliferation and naevogenesis driven by low-dose UVR that mimics mild sunburn.

As reported ${ }^{6},{ }^{\mathrm{V} 600 \mathrm{E}} \mathrm{BRAF}$ induced melanoma in $\sim 70 \%$ of mice at a median latency of 12.6 months. On average each mouse developed 0.9 tumours on its back (Fig 2a, 2b). When exposed to UVR, all V600E BRAF mice developed melanoma within 7 months at a median latency of 5.3 months and an average of $\sim 3.5$ (range 1-6) tumours each, $98 \%(59 / 60)$ of which were within the UVR-exposed area (Fig 2a-2c). UVR did not induce melanoma in non- ${ }^{\mathrm{V} 600 \mathrm{E}}$ BRAF mice (Fig. 2a). Thus, we concluded that UVR accelerated ${ }^{\mathrm{V} 600 \mathrm{E}} \mathrm{BRAF-}$ driven melanomagenesis.

The UVR and non-UVR tumours were asymmetrical, predominantly amelanotic and locally destructive (Fig. 2d, Extended Data Fig. 4a, 4b). They invaded the deeper subcutis, presented superficial naevi and pigmented cells and $\sim 50 \%$ ulcerated the overlying epidermis (Fig. 2e, 2f, Extended Data Fig. 4b). The tumour cells were atypical with dendritic/plump spindle morphology, and S100 and Hmb45/MelanA positive (Fig. 2e, 2f, Extended Data Fig. $4 \mathrm{c}-\mathrm{e}$ ). They were heterogeneous, highly mitotic (20 mitoses/10 high-power fields; 10 tumours analysed) and Ki-67 positive (26.4\%, range 10-50; Extended Data Fig. 4a, 4f).

Application of sunscreen (UVA superior, UVB SPF 50) 30 minutes before UVR exposure blocked induction of Trp53 and apoptosis in the epidermal keratinocytes (Extended Data Fig. 5a-c, Extended Data Table 1). These are largely UVB-driven responses ${ }^{7}$, but sunscreen 
also blocked UVA-induced epidermal hypertrophy and dermal thickening (Extended Data Fig. 5d). The cloth-protected and sunscreen-protected naevi were indistinguishable and sunscreen prevented UVR-driven skin darkening (Extended Data Fig. 6a, 6b). Thus sunscreen protected the epidermis from the immediate effects of UVA and UVB, but nevertheless all of the sunscreen-protected UVR-exposed ${ }^{\mathrm{V} 600 \mathrm{E}} \mathrm{BRAF}$-mice developed tumours within 15 months at a median latency of 7.5 months (Fig. 2a), a significant reduction compared to unprotected mice, but a significant increase compared to non-UVRexposed mice ( $\mathrm{p}=0.03$; Fisher Exact Test). Sunscreen protected mice developed $\sim 1.5$ tumours on their backs, again significantly fewer than unprotected mice, but significantly more than non-UVR-exposed mice (Fig. 2b, 2c). The sunscreen-protected melanomas presented similar histology to non-UVR and UVR-exposed melanomas (Fig. 2d-f, Extended Data Fig. 6c) and were more common on sunscreen-protected (14 tumours) than clothprotected (8 tumours) skin. Thus, sunscreen only partially prevented acceleration of ${ }^{\mathrm{V} 600 \mathrm{E}}$ BRAF-driven melanomagenesis by UVR.

Array comparative genome hybridisation revealed no significant differences in gross chromosomal aberrations or copy number variations between non-UVR and UVR melanomas (Fig. 3a), but the non-UVR melanomas presented significantly more (60\% vs. $13.3 \%$ ) recurrent copy number changes in melanoma oncogenes and tumour suppressors (MITF, BRAF, CCND1, CDKN2A; Fig. 3b; Supplementary Table 1) ${ }^{11}$. Whole exome sequencing (WES) revealed that UVR-exposed melanomas presented significantly more somatic single nucleotide variants (SNVs; median 6.16 [range 0.26-40.69] vs. 0.19 SNVs/Mb [range 0.02-2.16]; Fig. 3c; Supplementary Table 1) and a significantly higher proportion of $\mathrm{C}$ to $\mathrm{T}$ transitions (median $16.7 \%$ vs. $76.6 \%$ ) at the $3^{\prime}$ end of pyrimidine dimers than non-UVR tumours (Fig. 3d, 3e). This provides direct evidence of UVR-induced DNA damage ${ }^{12}$ and notably, although sunscreen significantly reduced the number of SNVs (median 0.25050 SNVs/Mb [range 0.08233 - 0.64432]) in UVR-exposed melanomas (Figure $3 \mathrm{c}$ and Supplementary Table 1), the tumours still presented a higher proportion of $\mathrm{C}$ to $\mathrm{T}$ transitions at the $3^{\prime}$ end of pyrimidine dimers than non-UVR exposed melanomas (Fig. 3d, $3 e$ ). Thus, sunscreen reduced overall mutation burden but did not completely block UVRinduced DNA damage.

We observed Trp53 mutations in 6 of 15 UVR-exposed melanomas, 1 of 11 sunscreenprotected UVR-exposed melanomas, but none of 15 non-UVR-exposed tumours (Fig 4a; Extended Data Table 3; Supplementary Table 1). We confirmed the Trp53 mutations (Fig. 4b; Extended Data Table 3a) and note that there were no significant differences in the number of SNVs in the UVR-exposed $\operatorname{Trp} 53$-mutant and $\operatorname{Trp} 53$-wild-type tumours (Extended Data Fig. 7a) suggesting that mutant $\operatorname{Trp} 53$ did not contribute to overall mutation burden. The mutations involved codons H39, S124, R245, R270, C272 and 6/7 were C to T transitions at the $3^{\prime}$ end of pyrimidine dimers (Extended Data Table 3a) supporting a direct role for UVR in induction of Trp53 mutations in melanoma. S124, R245, R270 and C272 are in the Trp53 DNA-binding domain and the corresponding residues (S127, R248, R273, C275) are mutated in human TP53 in melanoma (Extended Data Table 3a), producing deleterious effects that severely impair promoter-specific TP53-transcriptional activity (Extended Data Table 3b) ${ }^{13}$. R273 and R248 are human cancer mutation hotspots and 
produce proteins with dominant-negative and other gain-of-function activities ${ }^{14-16}$. Nterminal TP53 mutations are rare and H39 is not conserved, but the equivalent residue (Q38) is mutated in human cancer (Extended Data Table 3a). Notably, all of the mutant proteins accumulated in our mouse melanomas (Fig. 4c; Extended Data Table 3a), suggesting common functional consequences.

Two conditional-inducible mouse alleles for DNA-binding domain Trp53 mutants are available, $\operatorname{Tr} p 53^{L S L-R 172 H}$ and $\operatorname{Trp} 53^{L S L-R 270 H}$. These mutant proteins are functionally similar; they possess dominant-negative and gain-of-function activities, and they accumulate in tumours ${ }^{16}$. The $\operatorname{Trp} 53^{L S L-R 172 H}$ mice were readily available, so we generated heterozygous mice that expressed one copy of ${ }^{\mathrm{R} 172 \mathrm{H}} \mathrm{Trp} 53$ in their melanocytes $\left(\right.$ Trp $53^{+/ L S L-R 172 H} ;$ Tyr: $:$ CreERT2 $\left.{ }^{+/}\right)$. These mice did not develop melanoma, but when

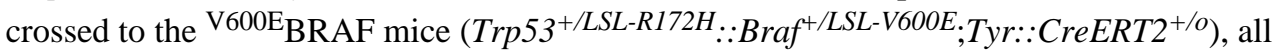
of the mice developed on average 6 tumours (range 2-10) on their backs within 3.5 months (Fig. 4d, 4e, 4f). The tumours presented the histopathological features of other ${ }^{\mathrm{V} 600 \mathrm{E}} \mathrm{BRAF}$ driven melanomas (Fig 4g, 4h; Extended Data Fig. 7b). Thus, as with deletion of one copy of the tumour suppressor Pten (Extended Data Fig. 7c) ${ }^{17}$, we show that mutant Trp53 accelerated BRAF-driven melanomagenesis ( $\mathrm{p}<0.0001$; Fisher Exact Test).

Finally, analysis of WES data from the Broad Institute ${ }^{18}$, The Cancer Genome Atlas (TCGA, https://tcga-data.nci.nih.gov/tcga/) and Yale ${ }^{19}$ revealed that TP53 mutations coincided with a higher proportion of $\mathrm{C}>\mathrm{T}$ transitions and a UVR signature in primary and metastatic humans cutaneous melanomas (Fig. 4i; Extended Data Fig. 7d; Extended Data Table 3c). Thus, in human and mouse melanomas, Trp53/TP53 mutations were associated with UVR-induced DNA damage, establishing that UVR accelerates melanomagenesis by targeting TP53/Trp53.

TP53 is a recognised UVR target in non-melanoma skin cancers, but is thought not to play a role in melanoma, because frequent $\mathrm{p} 14{ }^{\mathrm{ARF}}$ loss, stabilises HDM2, inactivating TP5 $3^{4}$. However, we show that $\sim 40 \%$ of mouse and $\sim 20 \%$ of human melanomas with evidence of UVR-induced DNA damage carried Trp53/TP53 mutations ${ }^{18}$. Trp53 gene deletions can cooperate with ${ }^{\mathrm{V} 600 \mathrm{E}} \mathrm{BRAF}$ to induce melanoma in mice and fish ${ }^{20,21}$, but in UVR-exposed melanomas TP53/Trp53 is mutated rather than lost, generating proteins with gain-offunction and dominant-negative activities ${ }^{14-16}$. This suggests a complex role for TP53/Trp53 in melanoma that cannot be accomplished if the protein is lost. Our data establish that TP53 is a bona fide UVR target in melanoma, providing insight into how UVR drives melanoma, albeit through mechanisms that have not yet been elucidated. Mutant TP53 drives tumourigenesis through processes inducing evasion of senescence, activation of autophagy, engagement of DNA-damage responses and oxidative stress pathway reactivation, but individual mutants influence these functions differently. Also, it was recently shown that UVR stimulates melanoma invasion, but the role of TP53 in this remains unknown ${ }^{22}$. Studies are required to elucidate the role of TP53 in melanoma and to identify other UVR targets in TP53/Trp53 mutant and TP53/Trp53 wild-type tumours. Intriguingly, recurrent UVR-signature mutations occur in RAC1, STK19 and PPP6C in melanoma, but their roles in melanomagenesis are currently unknown ${ }^{18,19}$. It is also unclear what induces ${ }^{\mathrm{V} 600 \mathrm{E}} \mathrm{BRAF}$ 
because it is not a UVR-signature mutation. Nevertheless, our data show that ${ }^{\mathrm{V} 600 \mathrm{E}} \mathrm{BRAF}$ expressing melanocytes are susceptible to UVR-driven naevogenesis and melanomagenesis.

In previous UVR melanoma models, embryonic mice constitutively expressed oncogenes, generally with loss of tumour suppressor genes, and the mice received a single burning dose of UVR 1-3 days after birth ${ }^{23,24}$. In this model melanoma is driven by inflammation mediated by IFN secreted by neonatal macrophages, and it does not accelerate V600E ${ }^{B R A F-}$ driven melanoma ${ }^{25}$. We expressed ${ }^{\mathrm{V} 600 \mathrm{E}} \mathrm{BRAF}$ at physiological levels in adolescent mice without manipulation of tumour suppressor genes and exposed the mice to repeated low doses of UVR. Thus we mimicked both somatic mutation acquisition and mild sunburn in humans, and our tumours were driven by acquired Trp53 mutations. Thus, we reveal two UVR melanoma pathways, one driven by inflammation in neonates and one driven by UVRinduced mutations in adults.

Finally, it is accepted that sunscreen protects against squamous cell carcinoma ${ }^{26}$, but controversy surrounds its' ability to protect against melanoma ${ }^{27-29}$. The US Environmental Protection Agency states that there is "no evidence that sunscreens protect you from malignant melanoma" (http://www.epa.gov/sunwise/doc/sunscreen.pdf), but we establish that sunscreen delayed UVR-driven melanoma in susceptible mice, validating public health campaigns that promote its use for melanoma. However, sunscreen did not safeguard against UVR completely and we advocate combining it with other sun avoidance strategies, particularly in at-risk individuals with BRAF-mutant naevi ${ }^{1,30}$.

\section{ONLINE-ONLY METHODS}

\section{Animal procedures}

All procedures involving animals were approved by the Animal Ethics Committees of the Institute of Cancer Research and Cancer Research UK Manchester Institute in accordance with National Home Office regulations under the Animals (Scientific Procedures) Act 1986 and according to the guidelines of the Committee of the National Cancer Research Institute $^{31}$. Tamoxifen (Sigma-Aldrich T5648) was freshly prepared in $100 \%$ ethanol. For genotyping, genomic DNA was prepared from tail biopsies and PCR was performed using the primers previously described ${ }^{6}$. Groups of $>10$ animals per cohort were based on historical precedent to provide statistically significant survival rates in Kaplan-Meier analysis. No randomisation or blinding were required. Animals were culled when tumour burden reached ethical limits, if the animals displayed signs of ill health or distress, or following a maximum of 24 months on study.

\section{V600EBRAF expression and UVR treatments}

V600E BRAF was expressed in female C57BL/6 mouse melanocytes by topical application of tamoxifen to the shaved backs of mice at $\sim 2$ months of age. A month later mice were anesthetized and the whole backs were shaved. Half of the shaved backs were covered vertically with a UVR proof cloth, to provide an internal control for non UVR-exposed skin. Each animal was treated with $160 \mathrm{~mJ} / \mathrm{cm} 2 \mathrm{UVA} / \mathrm{UVB}$ or $15 \mathrm{~J} / \mathrm{cm} 2 \mathrm{UVA}$ (Waldmann UV181 lamps fitted with UV 6 or UV A-1 tubes) each week for up to 6 months. For the 
sunscreen protection experiments, mice were treated with Sunsense Milk Sunscreen SPF50

(FDA standard 2,2 $\mathrm{mg} / \mathrm{cm} 2$,) by topical application $30 \mathrm{~min}$ before UVR exposure.

\section{Histology and immunohistochemistry}

For immunohistochemistry, samples were processed as described ${ }^{6}$. Briefly, sections were deparaffinized with xylene and hydrated with a series of graded alcohol washes. Sections were microwaved in citrate buffer $(\mathrm{pH}$ 6) for antigen retrieval and rinsed in PBS washes. Sections were blocked in 1\% BSA in PBS, incubated with 1:200 dilutions of anti-S100 (Dako), 1:100 dilutions of anti-Ki-67 (Santa Cruz), 1:200 dilutions of mouse anti-HMB45/ MelanA (Abcam), 1:100 Tp53 (Abcam) antibodies. Appropriate negative and positive controls for specificity of staining were included and images were captured using Leica DM4000 B LED automated upright microscope system.

\section{Naevi quantification}

For each animal, at least 3 samples of skin were collected. The skin sections were cut perpendicular to the longitudinal axis of the animals to span the exposed and non-exposed areas of the tamoxifen treated back. Two independent observers (observer 1 and 2, AV and $\mathrm{MC}$ respectively) performed naevi quantification. For each animal, naevi were quantified in 3 different skin sections, $1.8 \mathrm{~mm}$ width each of UVR-exposed and protected skin.

Significance was tested with the Wilcoxon Rank Sum Test. The size of the naevi was measured by a single observer recording the largest diameter for each nevus in $10.8 \mathrm{~mm}$ skin of UVR-exposed and non-exposed protected skin and significance tested using the U Mann Whitney Test. Four animals were scored.

\section{Tp53 quantification}

We quantified TP53 by scoring nuclear staining in 500 consecutive keratinocytes for each mouse in UVR exposed, protected, and UVR + sunscreen treated skin. Four animals were scored.

\section{Sunburn cell quantification}

We quantified sunburn cells in $1 \mathrm{~mm}$ of interfollicular basal keratinocytes for each mouse in UVR-exposed, protected, and UVR + sunscreen-treated skin. Four animals were scored.

\section{Immunofluoresence (IF)}

$3 \mathrm{~mm}$ sections of formalin fixed paraffin embedded (FFPE) material were used. Slides were dewaxed and antigen retrieval was performed using citrate buffer $\mathrm{pH} 6$ followed by blocking in PBS-Tween $0.1 \%+1 \%$ BSA for 15 min and overnight incubation with anti ki67 (Santa Cruz sc-7846) and S100 antibody (1:100 in PBS+1\%BSA). Antibody detection was performed using AlexaFluor-conjugated secondary antibodies (Invitrogen). Slides were counterstained with DAPI. Samples were analysed and pictures of the mid dermis were taken using a Leica confocal scanning microscope (Leica Microsystems). 


\section{Tumour analysis}

Mouse genomic DNA was prepared for aCGH and WES as described in Supplementary Information. 107 randomly selected SNVs were validated by Sanger sequencing to reveal a true positive rate of $93.5 \%$.

\section{WES and aCGH}

75 manually dissected sections of $20 \mu \mathrm{m}$ snap frozen tissue samples with an estimated tumour cell percentage of at least $80 \%$ were used for DNA extraction. Normal DNA was isolated from liver or kidney. DNA extraction was performed using a DNeasy blood and tissue kit (Qiagen). Quality assessment was performed by NanoDrop and Qubit. For aCGH, $2 \mu \mathrm{g}$ of normal and tumour DNA were hybridized to Nimblegen HX3 MM9 whole genome CGH arrays (100718_MM9_WG_CGH). Normalization, window averaging and segmentation were conducted using the standard Nimblegen aCGH analysis pipeline. The BioConductor package aCGH was used to summarise and plot copy number alterations. For WES, 15 tumours from UVR-exposed areas, 15 tumours from animals not exposed to UVR and 11 tumours from animals exposed to UVR and treated with sunscreen were included. Matched normal DNA samples from liver or kidney were obtained. DNA was extracted from frozen materials. Exome capture was performed using the Agilent SureSelect Mouse exome capture kit $3 \mu \mathrm{g}$ of gDNA according to the manufactures instructions. Sequencing was performed on Illumina GAIIx and HiSeq machines to produce 74bp paired end reads and quality control was conducted using FASTQC (http:// www.bioinformatics.babraham.ac.uk/projects/fastqc/). Reads were aligned to the NCBI m37 reference genome using BWA ${ }^{32}$ (version 0.5.9-r16; default parameters). Duplicate reads were marked by Picard (http://picard.sourceforge.net/index.shtml) and base quality score recalibration and local realignment around indels were performed using GATK $^{33}$ (version v1.2-24-g6478681). Pileup files of on-target high mapping quality bases (read mapping quality 230 ) were generated using Samtools ${ }^{34}$ (version 0.1.18) and somatic single nucleotide variants (SNVs) were called using Varscan ${ }^{35}$ (version v2.2.8) methods somatic (parameters -min-var-freq 0.08, -p-value 0.05, -min-avg-qual 20) and processSomatic at positions with coverage $\geq 10$ in the normal and tumour. Likely false positives were removed using predetermined filters ${ }^{35}$. The somatic mutational status of Trp53 in all tumour samples was also verified using Somaticsniper ${ }^{36}$. No mutations in Trp53 in the NON-UVR melanoma samples were identified by either method. Somatic variants were annotated by the Ensembl Variant Effect Predictor ${ }^{37}$ using Ensembl version 67 and variants present in dbSNP were excluded.

For Sanger sequencing validation, gDNA was amplified by PCR to confirm selected mutations. The products were directly sequenced using dye-terminator chemistry as previously described ${ }^{38}$. Sequences were visualized using Sequencher software. Primer sequences are available upon request. 107 randomly selected SNVs were validated by Sanger sequencing, to reveal a true positive rate of $93.5 \%$.

\section{Supplementary Material}

Refer to Web version on PubMed Central for supplementary material. 


\section{ACKNOWLEDGEMENTS}

This work was supported by Cancer Research UK (C107/A10433; C5759/A12328), the Wenner-Gren Foundations, Stockholm, Teggerstiftelsen (M.P.), and a FEBS Long-Term Fellowship (BS-L). We thank Garry Ashton of the Histology Units at the CRUK Manchester Institute for technical assistance and Dr Antony Young (Guy's, King's and St Thomas' School of Medicine, London) for helpful discussions. We would like to acknowledge the contribution of the melanoma specimen donors and research groups to The Cancer Genome Atlas.

\section{REFERENCES}

1. Whiteman DC, et al. Melanocytic nevi, solar keratoses, and divergent pathways to cutaneous melanoma. J Natl Cancer Inst. 2003; 95:806-812. [PubMed: 12783935]

2. Gilchrest BA, Eller MS, Geller AC, Yaar M. The pathogenesis of melanoma induced by ultraviolet radiation. N Engl J Med. 1999; 340:1341-1348. doi:10.1056/NEJM199904293401707. [PubMed: 10219070]

3. Yeh I, von Deimling A, Bastian BC. Clonal BRAF Mutations in Melanocytic Nevi and Initiating Role of BRAF in Melanocytic Neoplasia. J Natl Cancer Inst. 2013; 105:917-919. doi:10.1093/jnci/ djt119. [PubMed: 23690527]

4. Tsao H, Chin L, Garraway LA, Fisher DE. Melanoma: from mutations to medicine. Genes Dev. 2012; 26:1131-1155. doi:10.1101/gad.191999.112. [PubMed: 22661227]

5. Harrison SL, MacLennan R, Speare R, Wronski I. Sun exposure and melanocytic naevi in young Australian children. Lancet. 1994; 344:1529-1532. [PubMed: 7983952]

6. Dhomen N, et al. Oncogenic Braf induces melanocyte senescence and melanoma in mice. Cancer Cell. 2009; 15:294-303. doi:10.1016/j.ccr.2009.02.022. [PubMed: 19345328]

7. Ponten F, Berne B, Ren ZP, Nister M, Ponten J. Ultraviolet light induces expression of p53 and p21 in human skin: effect of sunscreen and constitutive p21 expression in skin appendages. The Journal of investigative dermatology. 1995; 105:402-406. [PubMed: 7665921]

8. Rudolph P, Tronnier M, Menzel R, Moller M, Parwaresch R. Enhanced expression of Ki-67, topoisomerase IIalpha, PCNA, p53 and p21WAF1/Cip1 reflecting proliferation and repair activity in UV-irradiated melanocytic nevi. Human pathology. 1998; 29:1480-1487. [PubMed: 9865836]

9. Pollock PM, et al. High frequency of BRAF mutations in nevi. Nature genetics. 2003; 33:19-20. doi:10.1038/ng1054. [PubMed: 12447372]

10. Thomas NE, et al. Number of nevi and early-life ambient UV exposure are associated with BRAFmutant melanoma. Cancer Epidemiol Biomarkers Prev. 2007; 16:991-997. doi: 10.1158/1055-9965.EPI-06-1038. [PubMed: 17507627]

11. Vultur A, Herlyn M. SnapShot: melanoma. Cancer Cell. 2013; 23:706-706. e701, doi:10.1016/ j.ccr.2013.05.001. [PubMed: 23680152]

12. Pfeifer GP, You YH, Besaratinia A. Mutations induced by ultraviolet light. Mutation research. 2005; 571:19-31. doi:10.1016/j.mrfmmm.2004.06.057. [PubMed: 15748635]

13. Kato $\mathrm{S}$, et al. Understanding the function-structure and function-mutation relationships of p53 tumor suppressor protein by high-resolution missense mutation analysis. Proceedings of the National Academy of Sciences of the United States of America. 2003; 100:8424-8429. doi: 10.1073/pnas.1431692100. [PubMed: 12826609]

14. Song H, Hollstein M, Xu Y. p53 gain-of-function cancer mutants induce genetic instability by inactivating ATM. Nature cell biology. 2007; 9:573-580. doi:10.1038/ncb1571. [PubMed: 17417627]

15. Willis A, Jung EJ, Wakefield T, Chen X. Mutant p53 exerts a dominant negative effect by preventing wild-type 53 from binding to the promoter of its target genes. Oncogene. 2004; 23:2330-2338. doi:10.1038/sj.onc.1207396. [PubMed: 14743206]

16. Olive KP, et al. Mutant p53 gain of function in two mouse models of Li-Fraumeni syndrome. Cell. 2004; 119:847-860. doi:10.1016/j.cell.2004.11.004. [PubMed: 15607980]

17. Dankort D, et al. Braf(V600E) cooperates with Pten loss to induce metastatic melanoma. Nature genetics. 2009; 41:544-552. doi:10.1038/ng.356. [PubMed: 19282848] 
18. Hodis E, et al. A landscape of driver mutations in melanoma. Cell. 2012; 150:251-263. doi: 10.1016/j.cell.2012.06.024. [PubMed: 22817889]

19. Krauthammer M, et al. Exome sequencing identifies recurrent somatic RAC1 mutations in melanoma. Nature genetics. 2012; 44:1006-1014. doi:10.1038/ng.2359. [PubMed: 22842228]

20. Patton EE, Zon LI. Taking human cancer genes to the fish: a transgenic model of melanoma in zebrafish. Zebrafish. 2005; 1:363-368. doi:10.1089/zeb.2005.1.363. [PubMed: 18248215]

21. Goel VK, et al. Melanocytic nevus-like hyperplasia and melanoma in transgenic BRAFV600E mice. Oncogene. 2009; 28:2289-2298. doi:10.1038/onc.2009.95. [PubMed: 19398955]

22. Bald $\mathrm{T}$, et al. Ultraviolet-radiation-induced inflammation promotes angiotropism and metastasis in melanoma. Nature. 2014; 507:109-113. doi:10.1038/nature13111. [PubMed: 24572365]

23. Noonan FP, et al. Neonatal sunburn and melanoma in mice. Nature. 2001; 413:271-272. doi: 10.1038/35095108. [PubMed: 11565020]

24. Luo C, et al. Loss of ARF sensitizes transgenic BRAFV600E mice to UV-induced melanoma via suppression of XPC. Cancer research. 2013; 73:4337-4348. doi: 10.1158/0008-5472.CAN-12-4454. [PubMed: 23650282]

25. Zaidi MR, et al. Interferon-gamma links ultraviolet radiation to melanomagenesis in mice. Nature. 2011; 469:548-553. doi:10.1038/nature09666. [PubMed: 21248750]

26. Green A, et al. Daily sunscreen application and betacarotene supplementation in prevention of basal-cell and squamous-cell carcinomas of the skin: a randomised controlled trial. Lancet. 1999; 354:723-729. doi:10.1016/S0140-6736(98)12168-2. [PubMed: 10475183]

27. Green AC, Williams GM, Logan V, Strutton GM. Reduced melanoma after regular sunscreen use: randomized trial follow-up. J Clin Oncol. 2011; 29:257-263. doi:10.1200/JCO.2010.28.7078. [PubMed: 21135266]

28. Goldenhersh MA, Koslowsky M. Increased melanoma after regular sunscreen use? J Clin Oncol. 2011; 29:e557-558. author reply e859, doi:10.1200/JCO.2011.35.5727. [PubMed: 21537031]

29. Planta MB. Sunscreen and melanoma: is our prevention message correct? J Am Board Fam Med. 2011; 24:735-739. doi:10.3122/jabfm.2011.06.100178. [PubMed: 22086817]

30. Viros A, et al. Improving melanoma classification by integrating genetic and morphologic features. PLoS Med. 2008; 5:e120. doi:10.1371/journal.pmed.0050120. [PubMed: 18532874]

\section{METHODS REFERENCES}

31. Workman P, et al. Guidelines for the welfare and use of animals in cancer research. British journal of cancer. 2010; 102:1555-1577. doi:10.1038/sj.bjc.6605642. [PubMed: 20502460]

32. Li H, Durbin R. Fast and accurate short read alignment with Burrows-Wheeler transform. Bioinformatics. 2009; 25:1754-1760. doi:10.1093/bioinformatics/btp324. [PubMed: 19451168]

33. McKenna A, et al. The Genome Analysis Toolkit: a MapReduce framework for analyzing nextgeneration DNA sequencing data. Genome Res. 2010; 20:1297-1303. doi:10.1101/gr.107524.110. [PubMed: 20644199]

34. Li H, et al. The Sequence Alignment/Map format and SAMtools. Bioinformatics. 2009; 25:20782079. doi:10.1093/bioinformatics/btp352. [PubMed: 19505943]

35. Koboldt DC, et al. VarScan 2: somatic mutation and copy number alteration discovery in cancer by exome sequencing. Genome Res. 2012; 22:568-576. doi:10.1101/gr.129684.111. [PubMed: 22300766]

36. Larson DE, et al. SomaticSniper: identification of somatic point mutations in whole genome sequencing data. Bioinformatics. 2012; 28:311-317. doi:10.1093/bioinformatics/btr665. [PubMed: 22155872]

37. McLaren W, et al. Deriving the consequences of genomic variants with the Ensembl API and SNP Effect Predictor. Bioinformatics. 2010; 26:2069-2070. doi:10.1093/bioinformatics/btq330. [PubMed: 20562413]

38. Turajlic S, et al. Whole genome sequencing of matched primary and metastatic acral melanomas. Genome Res. 2012; 22:196-207. doi:10.1101/gr.125591.111. [PubMed: 22183965] 

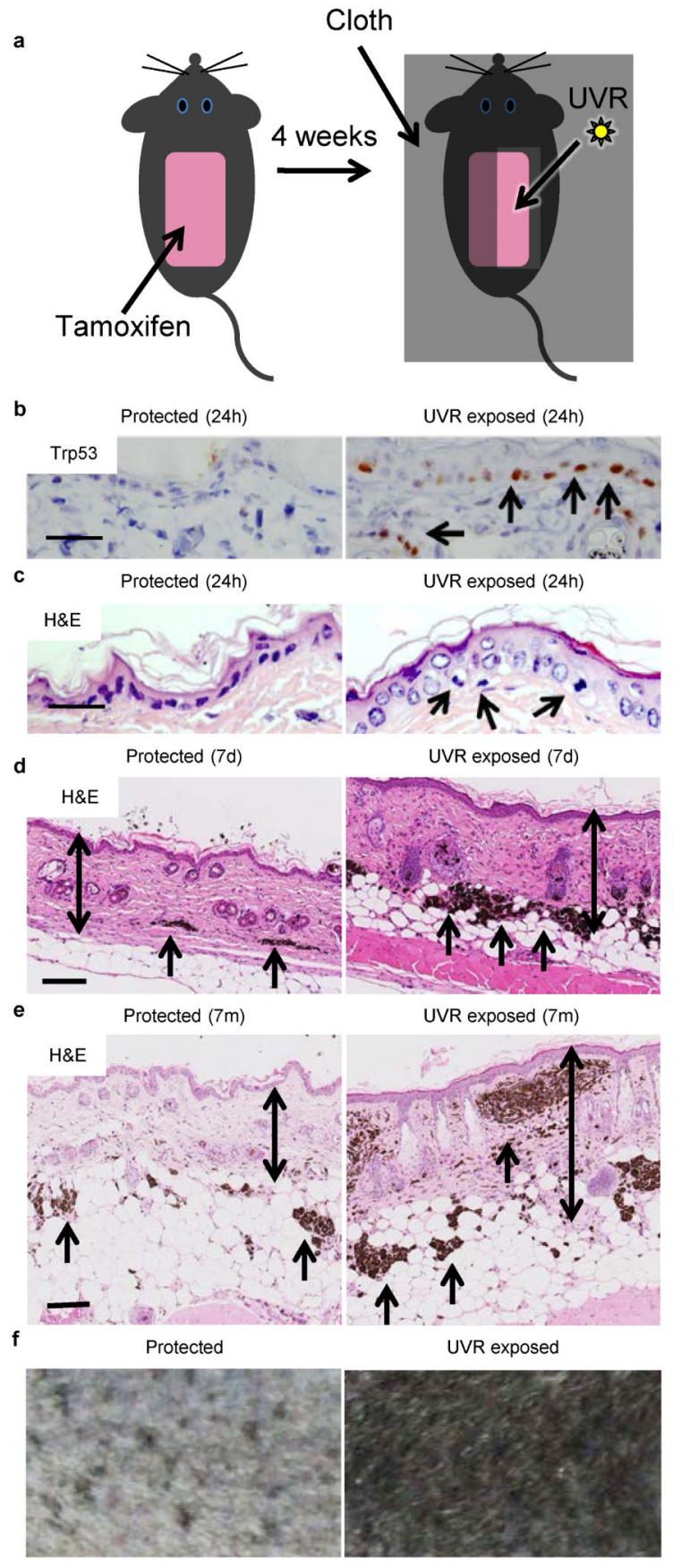

Figure 1. UV accelerates ${ }^{\mathrm{V} 600 \mathrm{E}}$ BRAF-driven naevogenesis

a. Experimental design.. b. Trp53 staining (arrows) in protected and UVR-exposed epidermis 24 hours (24h) after UVR. Bar: 50 $\mu$ m. 5 animals examined. c. Hematoxylin and eosin stained section (H\&E) of protected and UVR-exposed epidermis 24h after UVR.

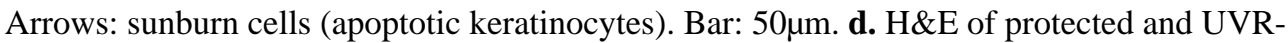
exposed epidermis 7 days (7d) after UVR. Arrows: dermal naevi; double-headed arrows: epidermal/dermal thickness. Bar: 500 $\mu$ m. e. H\&E of protected and UVR-exposed epidermis 
7 months (7m) after UVR. Arrows: dermal naevi; double-headed arrows: epidermal/dermal

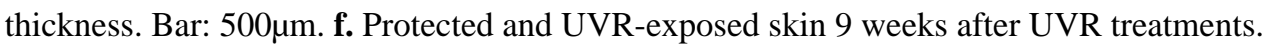


a

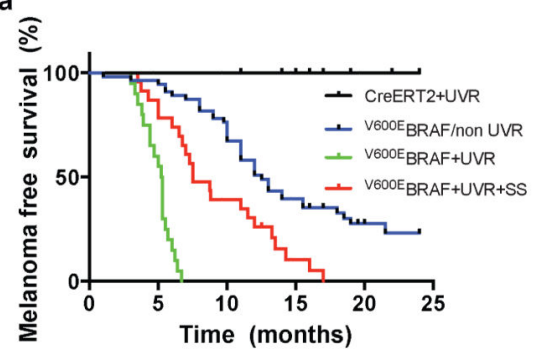

c

b

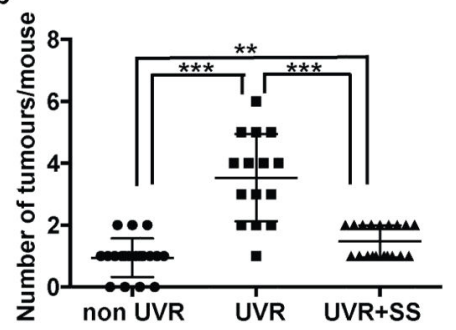

Protected UVR+SS

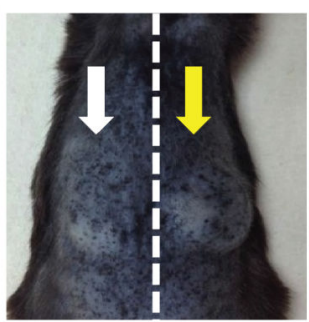

UVR

d

Non UVR

UVR+SS

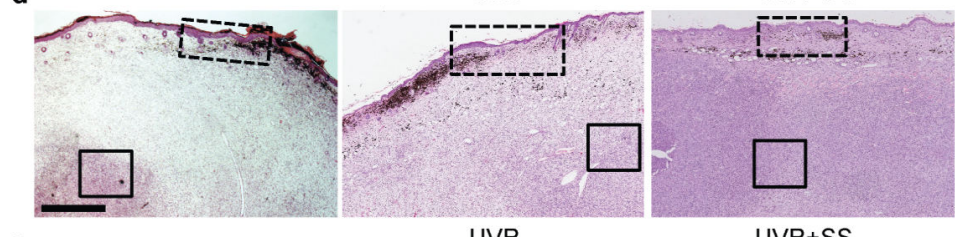

e

Non UVR

UVR

UVR+SS
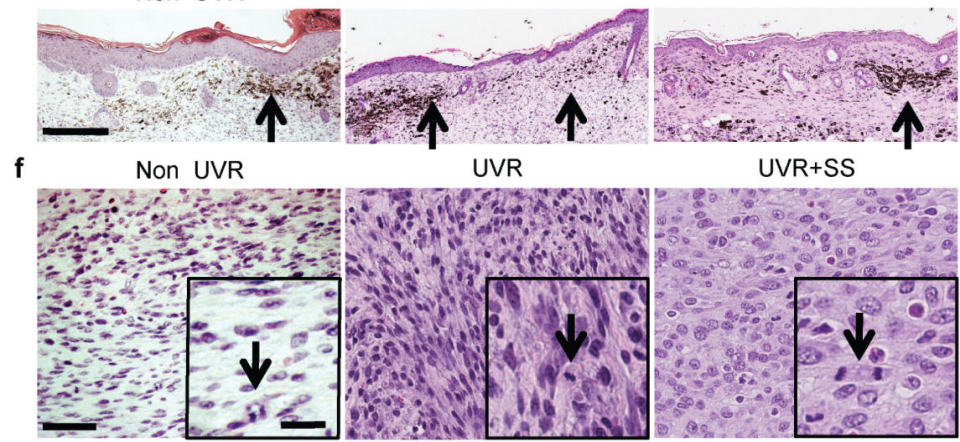

Figure 2. UVR accelerates BRAF ${ }^{\mathrm{V} 600}$-driven melanomagenesis

a. Kaplan-Meier showing melanoma-free survival in tamoxifen-treated UVR-exposed CreERT2 mice (CreERT2+UVR; $n=14)$; V600EBRAF mice ( ${ }^{\mathrm{V} 600 \mathrm{E}} \mathrm{BRAF} / \mathrm{non}$ UVR; $\mathrm{n}=55$ ); UVR-exposed V600E BRAF mice ( ${ }^{\mathrm{V} 600 \mathrm{E}} \mathrm{BRAF+UVR}$; $\left.=19\right)$ and UVR-exposed sunscreenprotected ${ }^{\mathrm{V} 600 \mathrm{E}} \mathrm{BRAF}$ mice $\left({ }^{\mathrm{V} 600 \mathrm{E}} \mathrm{BRAF}+\mathrm{UVR}+\mathrm{SS} ; \mathrm{n}=22\right)$. ${ }^{\mathrm{V} 600 \mathrm{E}} \mathrm{BRAF} /$ non-UVR vs. ${ }^{\text {V600E }}$ BRAF+UVR, $\mathrm{p}<0.0001$; ${ }^{\mathrm{V} 600 \mathrm{E}} \mathrm{BRAF+UVR}$ vs. ${ }^{\mathrm{V} 600 \mathrm{E}} \mathrm{BRAF}+\mathrm{UVR}+\mathrm{SS}, \mathrm{p}$ $<0.0001 ;{ }^{\mathrm{V} 600 \mathrm{E}} \mathrm{BRAF} / \mathrm{non}-\mathrm{UVR}$ vs. ${ }^{\mathrm{V} 600 \mathrm{E}} \mathrm{BRAF}+\mathrm{UVR}+\mathrm{SS}, \mathrm{p}=0.0003$; Log-rank Test. All tests are 2-sided. b. Median tumour numbers in V600E BRAF/non-UVR (nonUVR), ${ }^{\text {V600E }}$ BRAF+UVR (UVR) and V600E BRAF+UVR+SS (UVR+SS) mice. The error bars show mean \pm SD. $* * *$ p- $<0.0001$; ** p-<0.008 Wilcoxon Signed-Rank Test (WSRT). c. Photograph of UVR mouse 5.3 months after UVR and UVR+SS mouse 6.5 months after UVR. Yellow arrows: UVR tumours; white arrow: protected tumours. d. H\&E from nonUVR, UVR and UVR+SS mice. Bar: 0.5mm. e. Dashed-boxes from Fig 2d. Black arrows: 


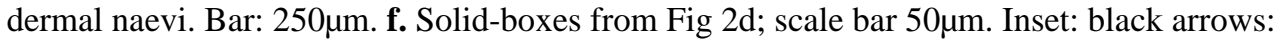
mitotic cells. Bar: $10 \mu \mathrm{m}$. 


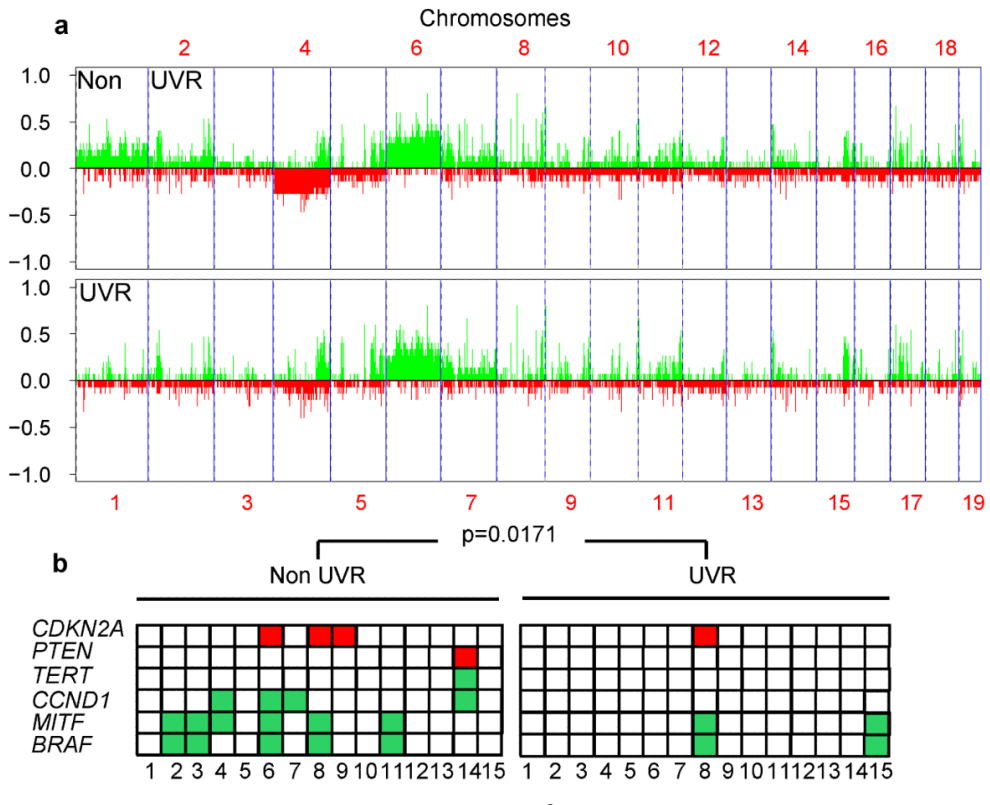

c

ns

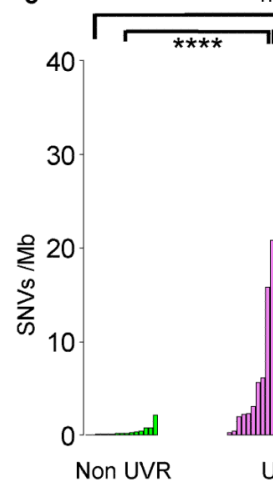

d
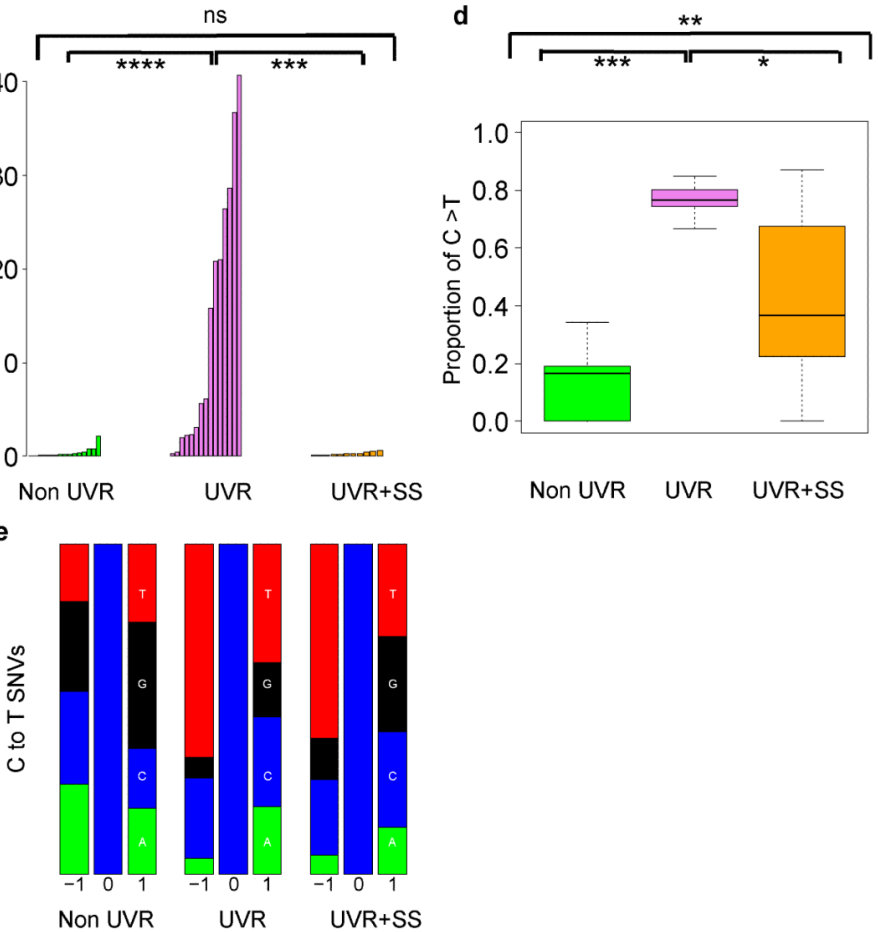

Figure 3. Genome analysis of UVR-driven melanomas

a. Somatic copy number alterations by aCGH in non-UVR and UVR tumours. b. Melanoma oncogene and tumour suppressor gene gains (green) and losses (red) by aCGH in non-UVR and UVR tumours. $\mathrm{p}=0.0171$; WRST. c. Somatic SNVs/Mb in non-UVR (green), UVR (violet) and UVR+SS (orange) tumours; ****: $\mathrm{p}=1.79 \mathrm{e}-06$; ***: $\mathrm{p}=1.165 \mathrm{e}-05$; ns: not significant $\mathrm{p}=0.6461$; WRST. d. Proportion of $\mathrm{C}>\mathrm{T}(\mathrm{G}>\mathrm{A})$ transitions at $3^{\prime}$ end of pyrimidine dimers in non-UVR, UVR and UVR+SS tumours. The error bars show the lowest data still within 1.5 IQR of the lowest quartile and highest data within 1.5 IQR of the 
upper quartile. $* * * \mathrm{p}<0.0001, * * \mathrm{p}=0.007698, * \mathrm{p}=0.02558$; WRST. e. Proportion of each nucleotide (A: green, C: blue, G: black, T: red) \pm 1 bp of all C to $\mathrm{T}$ ( $\mathrm{G}$ to A) transitions in non-UVR, UVR and UVR+SS tumours. 


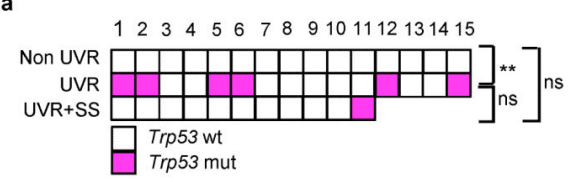

b
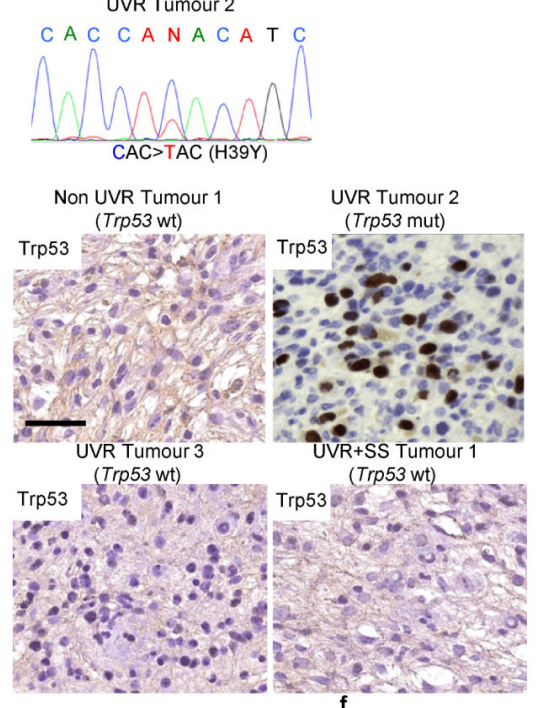

d
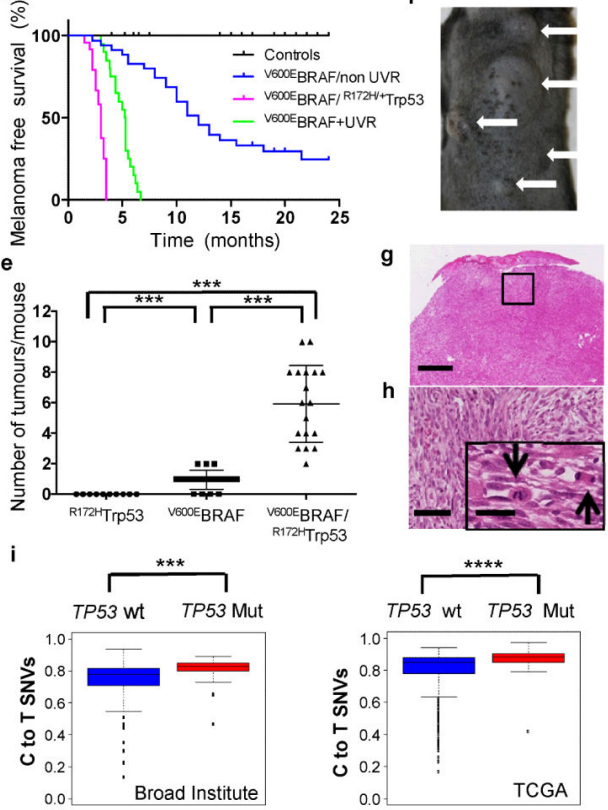

Figure 4. Mutant Trp53 accelerates ${ }^{\mathrm{V} 600 \mathrm{E}_{\text {BRAF-driven melanomagenesis }}}$

a. Trp53 mutations (wt: wild-type, white; mut: mutant, magenta) in non-UVR, UVR and UVR+SS tumours; ** p=0.017; Fisher Exact Test. b. Sanger sequencing confirming C>T, p.H39Y Trp53 mutation in UVR tumour 2. c. Trp53 immunostaining in non-UVR Tumour 1 (Trp53 wt), UVR Tumour 2 (Trp53 mut), UVR Tumour 3 (Trp53 wt), and UVR +SS

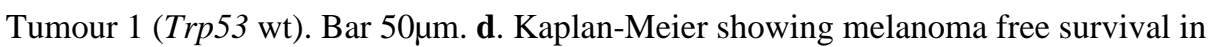
tamoxifen-treated CreERT2 and CreERT2/R172H Trp53 (control; n=42), ${ }^{\mathrm{V} 600 \mathrm{E}} \mathrm{BRAF} / \mathrm{non}$ $\operatorname{UVR}(\mathrm{n}=35),{ }^{\mathrm{V} 600 \mathrm{E}} \mathrm{BRAF} /{ }^{R 172 H} \operatorname{Trp53}(\mathrm{n}=23)$ and V600E BRAF+UVR $(\mathrm{n}=19)$ 
mice. ${ }^{\mathrm{V} 600 \mathrm{E}_{\mathrm{BRAF}} / \text { non-UVR vs. V600E BRAF/R172H }}$ Trp53, p $<0.0001$; ${ }^{\mathrm{V} 600 \mathrm{E}_{\mathrm{BRAF}}}{ }_{\mathrm{BRV}}$ vs. ${ }^{\mathrm{V} 600 \mathrm{E}_{\mathrm{BRAF}} /{ }^{\mathrm{R}} 172 \mathrm{H}} \mathrm{Trp53}$, $\mathrm{p}<0.0001$; Log-rank Test. e. Median tumour numbers in tamoxifen-treated CreERT2/ ${ }^{\mathrm{R} 172 \mathrm{H}} \mathrm{Trp} 53$ mice $\left({ }^{\mathrm{R} 172 \mathrm{H}} \mathrm{Trp} 53\right),{ }^{\mathrm{V} 600 \mathrm{E}_{\mathrm{BR}} \mathrm{RF} / \text { non-UVR }}$

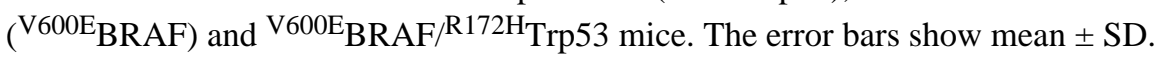
***p $<0.0001$, WRST. f. ${ }^{\mathrm{V} 600 \mathrm{E}} \mathrm{BRAF} /{ }^{\mathrm{R} 172 \mathrm{H}} \mathrm{Trp} 53$ mouse with multiple tumours (white arrows). g. $\mathrm{H} \& \mathrm{E}$ from a ${ }^{\mathrm{V} 600 \mathrm{E}_{\mathrm{BRAF}} /{ }^{\mathrm{R}} 172 \mathrm{H}} \mathrm{Trp} 53$ tumour. Bar $0.5 \mathrm{~mm}$. h. Boxed area from Fig. 4g. Bar: 250 $\mu$ m. Inset black arrows: mitotic cells. Bar: $5 \mu \mathrm{m}$. i. Proportion of $\mathrm{C}>\mathrm{T}$ (G>A) transitions in TP53 wild-type (wt) or mutant (mut) human melanomas from the Broad Institute ${ }^{18}(* * * \mathrm{p}=0.002$, WRST) and TCGA (https://tcga-data.nci.nih.gov/tcga/; $* * * * \mathrm{p}=1.79 \mathrm{E}-05$ ) datasets. The error bars show the lowest data still within 1.5 IQR of the lowest quartile and highest data within 1.5 IQR of the upper quartile. 